\title{
Investigating gating mechanisms of ion channels using temperature-resolved cryoEM
}

Harsh Bansia ${ }^{1}$, Claudio Catalano ${ }^{2}$, Zephan Melville ${ }^{3}$, Youzhong Guo ${ }^{2}$, Andrew R. Marks ${ }^{3}$ and Amedee des Georges ${ }^{1}$

${ }^{1}$ CUNY Advanced Science Research Center, New York, United States, ${ }^{2}$ Virginia Commonwealth University, Richmond, Virginia, United States, ${ }^{3}$ Columbia University, New York, United States

Technical advances in cryogenic electron microscopy (cryoEM) allow for high-resolution structural analysis of membrane proteins in near-native lipid environments $(1,2)$. Gating of several ion channels is temperature-correlated (3-5) yet structures are generally obtained at a single temperature with samples usually kept at low temperature $\left(4^{\circ} \mathrm{C}\right)$ resulting in sampling out important macromolecular conformations implicit in gating mechanism (6). Ryanodine receptors (RyRs) are homotetrameric 2.2 MDa calcium release channels located on sarco/endoplasmic reticulum (SR/ER) (Fig. 1A). Two isoforms, RyR1 and RyR2, are key elements in skeletal and cardiac muscle excitation-contraction coupling (E-C coupling) (7). E-C coupling controls muscle contraction by the tightly regulated release of $\mathrm{Ca} 2+$ from SR stores by RyR channels (Fig. 1A) (8). Abnormal RyR gating is responsible for muscle disorders including cardiac arrhythmias, heart failure, central core disease and malignant hyperthermia (7). E-C coupling is temperature sensitive and its thermosensitivity is correlated with temperature-induced triggering of RyR gating (4). Despite several recent high-resolution structures of RyR $(8,9)$, the molecular mechanism of RyR gating and its high thermal sensitivity are still not fully understood. Recent mathematical studies based on the electron-conformational model have accounted for the qualitative and quantitative effects of temperature on RyR gating (4). However, the mechanisms predicted by the electron-conformational model explaining temperature effects on the RyR gating (4) cannot be reconciled with available RyR structures as temperature was not included as one of the parameters in those Cryo-EM based structural studies and was far from the physiological temperature $\left(4^{\circ} \mathrm{C}\right.$ incubation prior to snap-freezing the samples). Mechanosensitive channel of small conductance (MscS) provides protection against hypo-osmotic shock in bacteria and its gating is modulated by membrane tension, voltage and cytoplasmic crowding (9) (Fig. 2A). Though established as a model protein for mechanosensation, recently MscS channels have been also proposed as a model protein for thermosensation (5). The tension in cell membrane changes dynamically in response to changes in temperature allowing temperature to indirectly modulate MscS activity (5) (Fig. 2B). Further, MscS channels may be intrinsically thermosensitive. Despite that, structural basis for intrinsic-extrinsic thermosensitivity of MscS has not been worked out so far.

All of the above considerations warrant a thorough structure-based investigation into the temperature effects on the gating mechanism of RyR and MscS (Figs. 1B and 2B). While a recent study has demonstrated the feasibility of multi-temperature cryoEM (11), temperature-resolved cryoEM is in infancy and its potential has not been realized for membrane proteins so far. We are using temperature as a thermodynamic variable in conjunction with cryoEM to probe the gating mechanism of RyR and MscS channels in unprecedented detail. Such information would provide a molecular framework for understanding temperature dependent gating of ion channels and a platform for the development of allosteric drugs that can restore normal RyR function.

CryoEM data collection and image processing of detergent purified RyRs and styrene maleic acid lipoprotein particles (SMALPs) purified MscS was done at different temperatures, ranging from $4{ }^{\circ} \mathrm{C}$ to 
$37{ }^{\circ} \mathrm{C}$. SMALPs are native cell-membrane based nanoparticles for purification and subsequent structural characterization of membrane proteins in near native conditions (2). Samples were incubated for a few minutes at a given temperature $\left({ }^{\circ} \mathrm{C}\right)$ (4-37) in the EM grid preparation chamber immediately prior to snap freezing the sample with liquid ethane allowing the molecules to sample the conformations accessible at that particular temperature. Extremely fast cryo-cooling will let the sample retain the ensemble-average structural properties at that temperature state. CryoEM data collection was carried out using standard procedures on NYSBC-SEMC Titan electron microscopes. Data was initially processed using Warp (12) and the resulting particle stack was subjected to 2D and 3D classification followed by refinements of reconstructed 3D maps in cryoSPARC (13).

Preliminary image processing of RyR1 cryoEM datasets showed considerable dynamics in the transmembrane (TM) region of the RyR1, which contains the $\mathrm{Ca} 2+$ permeation pathway and is the most important functional part of RyR1 (Fig. 1C). As can be seen in 2D class averages in Fig. $1 \mathrm{C}$, at $37{ }^{\circ} \mathrm{C}$ a fraction of the detergent-purified channels has a completely disordered TM domain. These results exemplified the instability of TM region of proteins outside of their lipid bilayer. On the contrary, 2D class averages from cryoEM data processing showed that SMALPs purified MscS retained intact TM region at high temperatures $\left(37^{\circ} \mathrm{C}\right)$ (Fig. 2C) highlighting the importance of biochemical and structural characterization of membrane proteins in native lipid environments and will be attempted for RyRs. Further, dynamics were observed in the TM region of MscS when structures obtained from different temperature datasets were superimposed to that of structure obtained at $4{ }^{\circ} \mathrm{C}$ (Fig. 2D) thereby demonstrating the temperature effects on the TM region of MscS implicated in its gating. However, molecules may exhibit continuous conformations relevant in their gating as opposed to discrete conformational heterogeneity sampled by traditional image classification methods (14). It therefore becomes necessary to sample the entire continuum of conformational states exhibited by the ion channel in order to provide faithful structural insights into its gating mechanism. This challenge will be addressed by using manifold embedding, an advanced geometric machine learning technique that maps the entire continuum of conformational states from the cryoEM 2D snapshots $(14,15)$. 
(A)

\section{Cytoplasm}

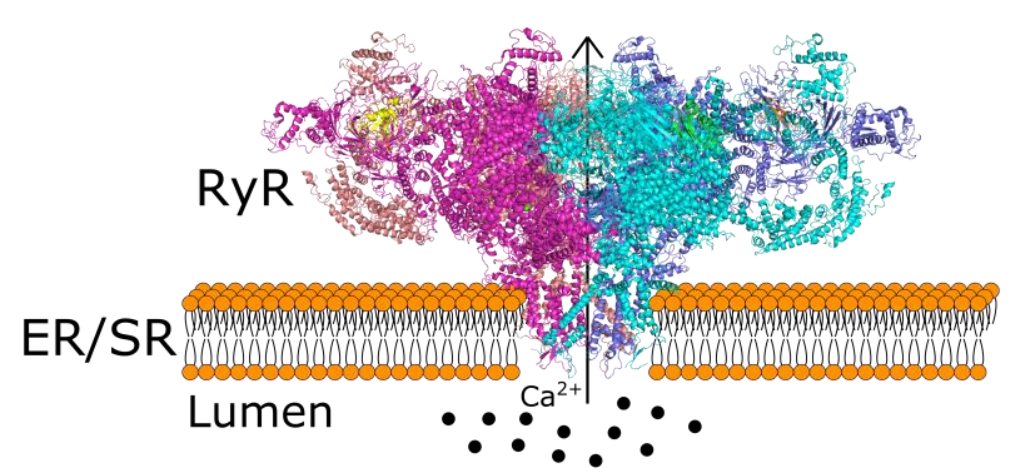

(B)
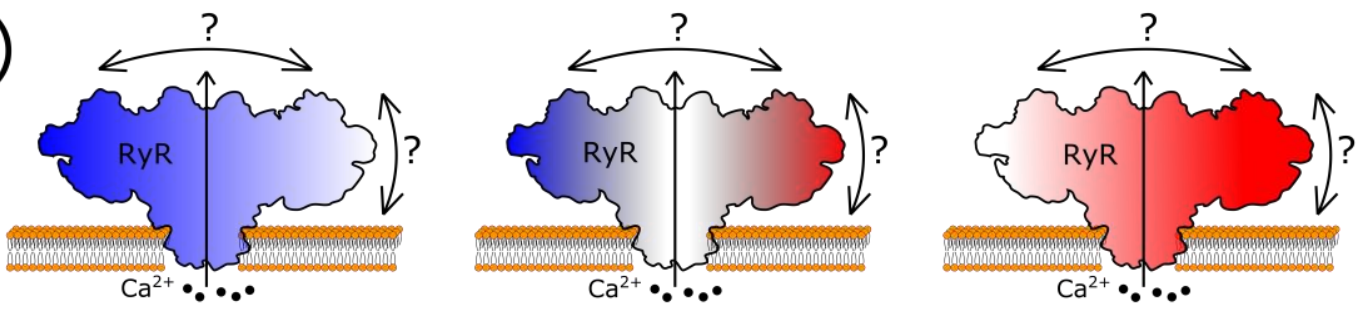

Temperature-dependent conformational states of RyR

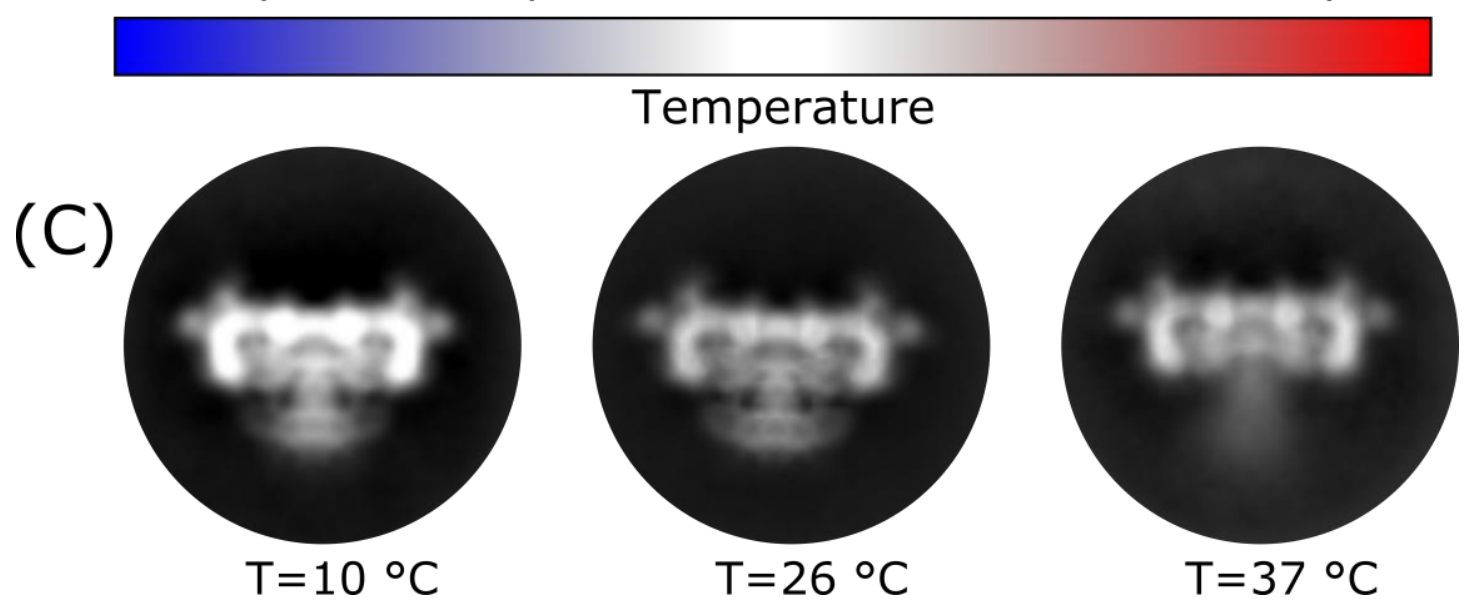

Figure 1. (A) Overall structure of Ryanodine receptor (RyR), homotetrameric $\sim 2.2 \mathrm{MDa}$ intracellular calcium $(\mathrm{Ca} 2+)$ release channel located on the sarco/endoplasmic reticulum (SR/ER). (B) Unknown conformational states of RyR associated with its temperature-dependent gating mechanism (C) 2D class averages of detergent purified RyR showing disordered transmembrane region at high temperatures. 
(A)

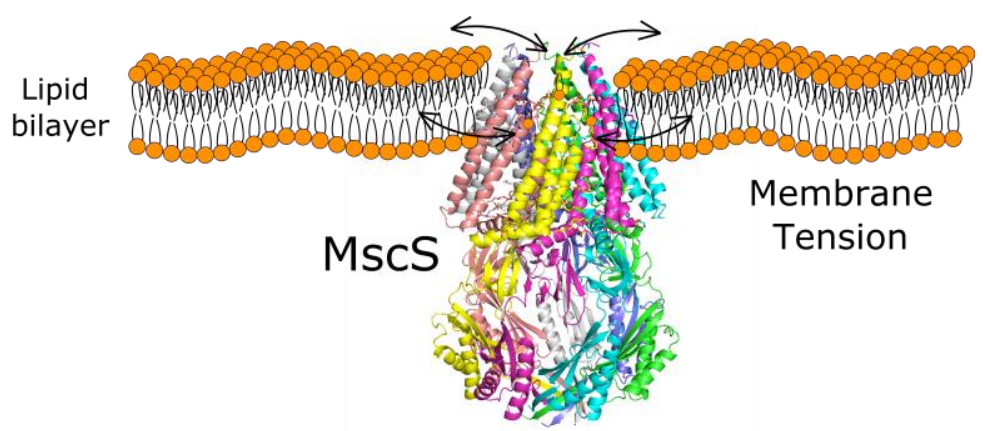

(B)
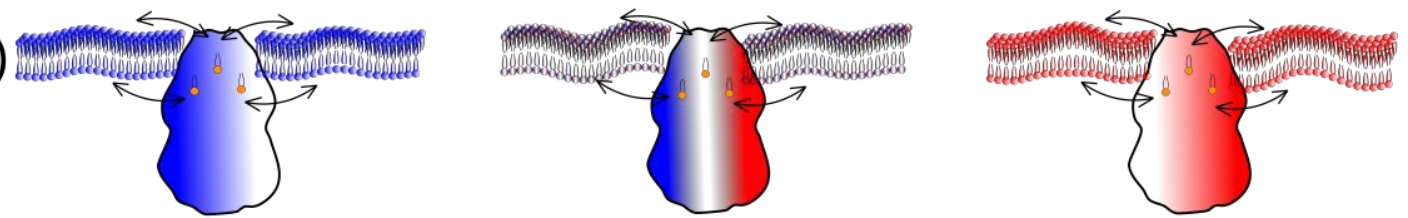

Temperature-dependent conformational states of MscS

(C)

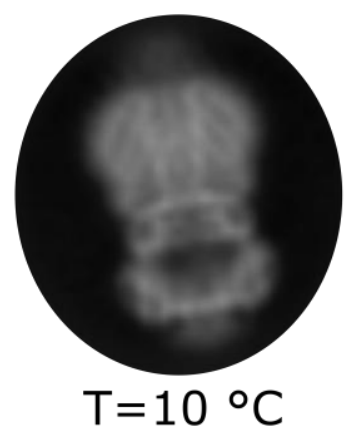

Temperature

(D)

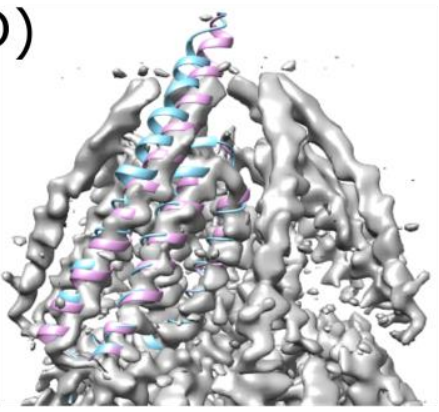

$\mathrm{T}=10^{\circ} \mathrm{C}$

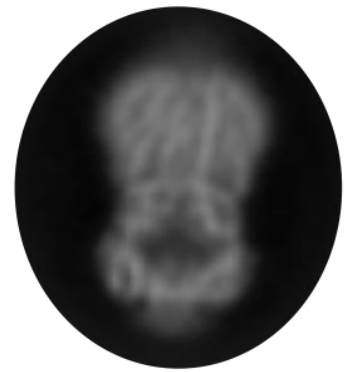

$\mathrm{T}=26^{\circ} \mathrm{C}$

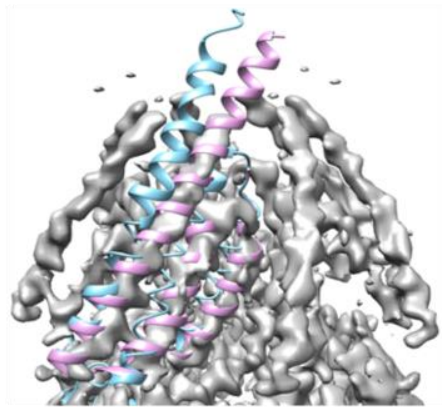

$\mathrm{T}=18^{\circ} \mathrm{C}$

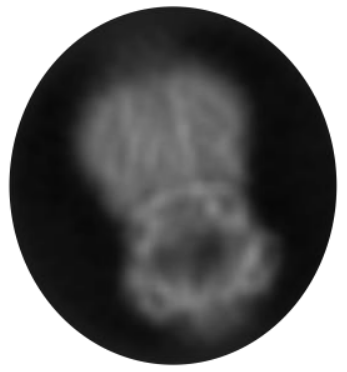

$\mathrm{T}=37^{\circ} \mathrm{C}$

Figure 2. (A) Overall structure of mechanosensitive channel of small conductance (MscS) with its gating modulated by membrane tension (B) Unknown conformational states of $\mathrm{MscS}$ associated with its temperature-dependent gating mechanism (C) 2D class averages of SMALPs purified MscS showing intact transmembrane region at high temperatures. (D) Observed dynamics in the TM region of MscS when structures obtained from different temperature datasets (pink helices) were superimposed to that of structure obtained at $4{ }^{\circ} \mathrm{C}$ (blue helices). 


\section{References}

heng Y. Membrane protein structural biology in the era of single particle cryo-EM. Curr Opin Struct Biol, 52, 2018.

1. Qiu W, et al. Structure and activity of lipid bilayer within a membrane-protein transporter. Proc Natl Acad Sci U S A, 115, 2018.

2. Chowdhury S, et al. A molecular framework for temperature-dependent gating of ion channels. Cell, $158,2014$.

3. Moskvin AS, et al. The temperature effect on cardiac ryanodine receptor gating and conductance: Mathematical modeling. BIOPHYSICS 61, 2016.

4. Koprowski P, et al. Negative and positive temperature dependence of potassium leak in MscS mutants: Implications for understanding thermosensitive channels. Biochim Biophys Acta, 1848, 2015.

5. Mehra R, et al. Cryo-temperature effects on membrane protein structure and dynamics. Phys Chem Chem Phys, 22, 2020.

6. Santulli G, et al. Physiology and pathophysiology of excitation-contraction coupling: the functional role of ryanodine receptor. J Muscle Res Cell Motil, 38, 2017.

7. Ogawa $\mathrm{H}$, et al. Regulatory mechanisms of ryanodine receptor/Ca ${ }^{2+}$ release channel revealed by recent advancements in structural studies. J Muscle Res Cell Motil, 2020.

8. des Georges A, et al. Structural Basis for Gating and Activation of RyR1. Cell, 167, 2016.

9. Naismith JH, Booth IR. Bacterial mechanosensitive channels-MscS: evolution's solution to creating sensitivity in function. Annu Rev Biophys, 41, 2012.

10. Chen CY, et al. Temperature-Resolved Cryo-EM Uncovers Structural Bases of TemperatureDependent Enzyme Functions. J Am Chem Soc, 141, 2019.

11. Tegunov D \& Cramer P. Real-time cryo-electron microscopy data preprocessing with Warp. Nat Methods, 16, 2019.

12. Punjani A, et al. cryoSPARC: algorithms for rapid unsupervised cryo-EM structure determination. Nat Methods, 14, 2017.

13. Frank $\mathbf{J} \&$ Ourmazd A. Continuous changes in structure mapped by manifold embedding of singleparticle data in cryo-EM. Methods, 100, 2016.

14. Dashti A, et al. Retrieving functional pathways of biomolecules from single-particle snapshots. Nat Commun, 11, 2020. 